\title{
CEREU
}

DOI:10.18605/2175-7275/cereus.v9n1p.57-75

\section{SATISFAÇÃO E TRABALHO NA ADMINISTRAÇÃO PÚBLICA FEDERAL: UMA INVESTIGAÇÃO COM SERVIDORES DO ENSINO SUPERIOR}

\author{
MATTOS, Carlos André Corrêa de ${ }^{1}$ \\ VIDAL, Josep Pont ${ }^{2}$ \\ LIRA, Monalisa da Silveira ${ }^{3}$ \\ COSTA, Nilson Luiz ${ }^{4}$ \\ ABUD, Glenda Maria Braga ${ }^{5}$
}

RESUMO

O objetivo deste artigo é analisar a satisfação com o trabalho no serviço público. A investigação foi feita na forma de pesquisa de campo, em uma instituição federal de ensino superior (IFES), no estado do Pará. A pesquisa, exploratória e descritiva, contou com 65 entrevistados em amostra não probabilística por acessibilidade. $O$ tratamento de dados utilizou a estatística descritiva e paramétrica (teste T). $\mathrm{O}$ instrumento de coleta de dados foi desenvolvido por Quijano e Navarro (1999), e os resultados permitem afirmar que há evidências

1Doutor em Ciências Agrárias pela Universidade Federal Rural da Amazônia (UFRA) professor permanente do Programa de Mestrado Profissional em Gestão Pública do Núcleo de Altos Estudos Amazônicos (NAEA).E mail para correspondência: carlosacmattos@hotmail.com

2Doutor em Sociologia Política na Universidad de Barcelona (1997). Professor adjunto e pesquisador do Núcleo de Altos Amazônicos (UFPA).

3Administradora, técnica administrativa da Universidade Fedral do Pará

4Doutor em Ciências Agrárias pela Universidade Federal Rural da Amazônia (2012). Coordenador do Programa de PósGraduação em Agronegócios (PPGAGR/UFSM).

5Mestranda em Meio Ambiente e Desenvolvimento Urbano da Universidade da Amazônia (UNAMA). 
significativas da presença de satisfação com o trabalho no local da pesquisa, com destaque para aspectos como estabilidade, relacionamento interpessoal e oportunidades de capacitação e desenvolvimento profissional. Intermediariamente, posicionaram-se questões relacionadas com instalações, equipamentos e benefícios sociais. Não foram identificadas diferenças significativas na satisfação com o trabalho entre as categorias investigadas.

Palavras-chave: Auditoria do Sistema Humano (ASH). Instituições Federais de Ensino Superior. Gestão de Pessoas.

\section{SATISFACTION AND WORK IN THE FEDERAL PUBLIC ADMINISTRATION: AN INVESTIGATION WITH HIGHER EDUCATION WORKERS}

\section{ABSTRACT}

The purpose of this article is to analyze satisfaction with work in the public service. The research was done trough field research, in a federal institution of higher education (IFES), in the state of Pará. The exploratory and descriptive research counted on 65 interviewees in a non-probabilistic sample for accessibility. The data treatment used the descriptive and parametric statistics ( $T$ test). The data collection instrument was developed by Quijano and Navarro (1999), and the results allow to affirm that there is significant evidence of satisfaction with work at the research site, with emphasis on aspects such as stability, interpersonal relationships and training opportunities and professional development. Intermediately, issues related to facilities, equipment and social benefits were posed. No significant differences in job satisfaction were identified among the categories investigated.

Key words: Human System Audit. Federal Institutions of Higher Education. management. 


\section{INTRODUÇÃO}

A realidade da sociedade moderna revelou profundos anacronismos da administração pública e desencadeou processos que culminaram com uma percepção diferente do que a sociedade espera dos serviços públicos. A busca pela excelência na gestão, combinada com uma atuação mais criativa $e$ adaptativa dos servidores públicos, colocou em xeque a rigidez e a racionalidade prescritiva dos manuais de normas e procedimentos, e alterou definitivamente a percepção puramente tecnicista do trabalho. Esse processo desencadeou mudanças nas estratégias de gestão de pessoas em organizações públicas (MELLO; AMANCIO FILHO, 2009).

Contudo, apesar dos avanços recentes, a legislação brasileira ainda carece de uma política de recursos humanos que seja de fato alinhada com as necessidades de suas organizações públicas (DUTRA, 2013; PINTO; SILVA, 2015; TEIXEIRA; SALOMÃO, 2013). Para cumprir efetivamente sua função, a gestão de pessoas deve ultrapassar os aspectos exclusivamente procedimentais, como planejamento e controle dos servidores públicos e o e políticas de recursos humanos, para incentivar uma participação mais efetiva dos servidores, de tal maneira que resulte na melhoria do trabalho e possa estimular uma atuação proativa, que culmine na convergência das estratégias em ações organizacionais (MELLO; AMANCIO FILHO, 2009).

Nesse contexto, compreender a gestão de pessoas sob uma perspectiva estratégica é uma forma privilegiada de possibilitar uma integração mais sinérgica entre os trabalhadores e as instituições, que favoreça a criação de valor e contribua efetivamente para uma relação de harmonia entre o desenvolvimento organizacional e das pessoas que as constituem. Esse processo envolve, entre outros aspectos, questões relacionadas com a satisfação com o trabalho. Isso posto, este estudo se concentrou em investigar a satisfação com o trabalho entre servidores de uma instituição de ensino superior, envolvendo tanto técnicos administrativos, quanto professores, e para isso, adotou como objetivo analisar a satisfação com o trabalho entre os servidores de uma instituição pública federal de ensino superior. 
Apesar de Meleiro e Siqueira (2005) advertirem que a conceituação de satisfação com o trabalho ainda carecer de consenso, Robbins (2014, p.65) compreende satisfação com o trabalho como a "atitude geral de uma pessoa em relação ao trabalho que realiza", e como consequência, ela promove atitudes positivas, sendo verdadeira também a interpretação contrária, ou seja, a insatisfação com o trabalho estimula atitudes negativas no trabalhador com relação às tarefas que realiza e para com a organização em que trabalha.

A satisfação com o trabalho é, portanto, um estado de ânimo de caráter eminentemente pessoal, mutável e dinâmico por natureza, no qual o indivíduo subjetivamente avalia as condições de sua atividade laboral, resultando em estados de alegria e de desprazer, sendo, ambos, sentimentos do mesmo fenômeno (LOCKE, 1976; FRASER, 1996).

A satisfação como trabalho tem sido o foco de diversos estudos (SANT'ANNA, MORAES, KILIMNIK, 2002; MARQUEZE; MORENO, 2005; MILAN; TREZ, 2005) que reforçam o interesse da academia pela temática. Nessa perspectiva, Marqueze e Moreno (2005) destacam a atenção especial que os estudos têm dedicado para a relação emocional do trabalhador com a atividade laboral (DAVIS, 2013). Ainda nesse contexto, Campbell, Converse e Rodgers (1976) já afirmavam que $25 \%$ da variabilidade da satisfação total na vida adulta advinham da satisfação com o trabalho, e Hapton (1992) justificava o interesse pela satisfação com o trabalho, considerando a grande quantidade de pesquisas sobre a temática, que, na década de 1990, já ultrapassava 2000 estudos nos 20 anos anteriores.

Mais contemporaneamente, Marques, Borges e Reis (2016), ao investigarem satisfação com o trabalho e mudança organizacional na administração pública, constataram que servidores mais abertos à mudança são mais satisfeitos com o trabalho.

Brum et al. (2014), ao buscarem relações entre satisfação com o trabalho e socialização, também em instituições públicas, encontraram complementariedade entre os dois construtos. Internacionalmente, Davis (2013), contrariando estudos anteriores, encontrou associação entre participação sindical e satisfação com o trabalho, e Kjeldsen e Hansen 
(2016), igualmente no setor público, reforçaram a relação positiva entre motivação e satisfação no trabalho.

Desta forma, os estudos mostram que, apesar de a satisfação com o trabalho não ser uma inquietude recente, ela continua a despertar o interesse acadêmico, especialmente na administração pública, o que se justifica por um amplo conjunto de aspectos, mas, destacadamente, pela necessidade de prover melhores serviços públicos, pelo interesse que a carreira pública desperta no mercado de trabalho e pelas mudanças enfrentadas pelas organizações do setor.

Com isso, essa investigação se concentrou em responder ao questionamento: quais elementos da relação laboral contribuem para a satisfação com o trabalho no serviço público?

Kjeldsen e Hansen (2016) destacam que, apesar de os estudos revelarem elevada satisfação no trabalho na administração pública, ainda são comuns nessas organizações aspectos fortemente prejudiciais à satisfação, como, por exemplo, processos muito burocráticos, hierarquia rígida e objetivos pouco definidos.
Desta forma, assumindo essas características como premissas, foram estabelecidas as seguintes hipóteses para esta investigação:

H1 - Não se pode afirmar que os servidores públicos estejam satisfeitos com 0 trabalho na instituição pesquisada.

H2- Não se pode afirmar que existe diferença na satisfação com o trabalho entre técnicos administrativos e professores na instituição.

Nesse contexto, estudar a satisfação com o trabalho se destaca pelo trabalho ser uma das principais atividades humanas e, entre tudo o que se pode realizar ao longo da vida, ele se evidencia entre as atividades mais relevantes, tanto pelo tempo a ele dedicado, quanto pela sua participação na formação de riqueza e manutenção da sociedade. Por esses e outros motivos, as pessoas passam grande parte de suas vidas ou no local de trabalho, ou fazendo tarefas a ele relacionadas. O trabalho, mais que uma forma de prover o sustento financeiro pessoal, é uma maneira privilegiada para obter realização, crescimento profissional, relacionar-se com outras pessoas, desenvolver habilidades, obter reconhecimento e uma forma de se integrar à sociedade. 


\section{MATERIAIS E MÉTODOS}

A pesquisa é classificada, conforme Gil (2014), como um estudo exploratório e descritivo, de abordagem quantitativa, na forma de pesquisa de campo, com amostragem não probabilística por acessibilidade. Essa forma de investigação possibilita a compreensão inicial do fenômeno e sua descrição, sem que, obrigatoriamente, viabilize sua explicação, que, para ser feita, demandaria outras técnicas de pesquisa.

Tendo como referência técnicas de estatísticas para tratar os dados e elucidar as hipóteses, a investigação privilegiou a medição e a quantificação para a obtenção dos objetivos, estando, entre as técnicas selecionadas, a distribuição de frequência, as medidas de tendência central (média), dispersão (desvio padrão) e os testes paramétricos, especificamente o teste $\mathrm{T}$, empregado na forma de uma amostra (one sample T-Test) e de duas amostras independentes, possibilitando, assim, atender as hipóteses da investigação.

A técnica de amostragem, por ser não probabilística, não permite o controle do erro amostral, portanto, não possibilita estender os resultados à população, limitando os achados à amostra estudada. Mesmo assim, essa técnica é adequada a pesquisas de caráter exploratório, atendendo as propostas estabelecidas neste estudo. A pesquisa de campo ocorreu no Instituto de Ciências Biológicas (ICB) da Universidade Federal do Pará (UFPa) e contou com a participação voluntária de 65 (n) servidores, dos 254 (N) lotados no instituto. A composição da amostra foi formada por 34 (n1) professores e 31 (n2) técnicos administrativos.

O instrumento de coleta de dados foi o questionário, desenvolvido e validado por Quijano e Navarro (1999), identificado como Auditoria do Sistema Humano (ASH), com o recorte nas variáveis que mensuravam a satisfação com o trabalho, tendo sido utilizada a versão traduzida e adaptada ao português, apresentada em Bertoldo (2006) e Barboza (2008). O questionário foi organizado em duas seções, a primeira teve a finalidade de investigar o perfil socioeconômico dos servidores e a segunda, com 17 questões, concentrou-se especificamente na avaliação da 
satisfação com 0 trabalho. As respostas foram formuladas em escala de Likert com opções de respostas que variaram entre 1 e 5, sendo: 1 para totalmente insatisfeito, 2 para parcialmente insatisfeito, 3 para nem satisfeito, nem insatisfeito, 4 para parcialmente satisfeito e 5 para totalmente satisfeito.

Os questionários incluíram o Termo de Consentimento Livre e Esclarecido (TCLE), conforme os padrões éticos de pesquisa com seres humanos. A fidedignidade do questionário foi mensurada pelo coeficiente alpha de Cronbach, evidenciando boa consistência interna, uma vez que o coeficiente obteve valor de 0,873 , superior ao valor recomendado por Hair et al. (2009), que consideram índice acima de 0,600 satisfatório para estudos exploratórios.

A hipótese de avaliação geral da satisfação com o trabalho $\left(\mathrm{H}_{1}\right)$ foi testada com a utilização do teste $T$ para uma amostra. A segunda hipótese, baseada na ausência de diferenças significativas na satisfação $\left(\mathrm{H}_{2}\right)$ de técnicos administrativos (n2) e professores ( $\mathrm{n} 1)$, foi testada com o teste $T$ na variação para duas amostras independentes.
A aplicação do teste ocorreu pelas médias amostrais. Os testes de hipóteses com base na média são empregados sempre que o pesquisador deseja testar afirmações relacionadas com parâmetros populacionais (BRUNI, 2013), e o teste $T$ é o teste paramétrico mais utilizado com esse objetivo (MALHOTRA, 2011).

Malhotra (2011) destaca que, para a utilização do teste $\mathrm{T}$, devem ser observados três prerrequisitos: presença de variáveis pelos menos na escala intervalar; (2) normalidade da distribuição amostral; e homogeneidade na variância populacional.

Barbeta (2014), ao apresentar os prerrequisitos comuns a todos os tipos de testes de hipóteses, destaca a necessidade de estabelecer, a priori, tanto as hipóteses do estudo, quanto a regra de decisão.

Desta forma, para atender a esse conjunto de prerrequisitos, 0 tratamento de dados foi iniciado pela verificação do tipo das variáveis e sua adequação ao teste. Esse aspecto foi atendido, uma vez que a escala de Likert é uma escala intervalar, em seguida, com as hipóteses estabelecidas, assim como os 
parâmetros dos testes. Foram avaliadas a normalidade da distribuição amostral (Shapiro-Wilks) e a homogeneidade das variâncias (teste F). Isso posto, foram

\section{RESULTADOS E DISCUSSÃO}

A amostra foi formada predominantemente por servidores do sexo feminino: $60,00 \%$ (39) dos entrevistados se posicionaram nesse estrato, $46,15 \%$ (30) dos entrevistados tinham entre 30 e 40 anos e $52,30 \%$ (34) se declararam casados. Quanto ao vínculo laboral, $92,30 \%$ (60) da amostra se enquadrava na categoria de estatutários regidos pelo sistema de Regime Jurídico Único (RJU), portanto, estáveis. Quanto ao salário,

\subsection{ANÁLISE DESCRITIVA:}

A satisfação dos servidores, Tabela 1, foi avaliada pelas 17 assertivas do modelo ASH (NAVARRO; QUIJANO, 1999), e como destaca Bertoldo (2006), a satisfação com o trabalho no modelo ASH é fundamentada pelas teorias motivacionais. Nesse contexto, o tratamento descritivo revelou alta satisfação com o trabalho, uma vez determinados a regra de decisão e o nível de significância do teste $(\alpha=0,05)$ para determinar a região crítica de aceitação e rejeição de $\mathrm{H} 0$.

os servidores se dividiram principalmente em dois estratos: $35,38 \%$ (23) declararam faixa salarial entre $R \$ 2.675,00$ e $R \$ 4.861,00$ por mês e outros $35,38 \%$ (23) declararam salário mensal entre $R \$ 4.862$ e $R \$$ $9.897,00$. A escolaridade da amostra foi elevada, uma vez que $83,07 \%$ (54) dos entrevistados tinham nível superior completo e $50,76 \%$ (33) eram titulados como doutores.

que $94,11 \% \quad$ (16) das variáveis apresentaram médias superiores ao ponto intermediário da escala $(3,00)$. Esse comportamento resulta da opção da maioria dos entrevistados pelos estratos superiores da escala, formados pelas alternativas parcialmente satisfeito $(4,00)$ e totalmente satisfeito $(5,00)$. A elevada satisfação com 0 trabalho foi 
confirmada posteriormente pelo teste $\mathrm{T}$ para uma amostra que possibilitou rejeitar a $\mathrm{H} 1$ e aceitar que existem evidências estatísticas para afirmar que os servidores estão satisfeitos com o trabalho no local da pesquisa.

Tabela 1 - Satisfação com o trabalho

\begin{tabular}{|c|c|c|c|c|c|c|}
\hline \multirow{2}{*}{\multicolumn{2}{|c|}{ Variáveis }} & \multicolumn{3}{|c|}{ Satisfação (em \%) } & \multirow{2}{*}{ Med. } & \multirow{2}{*}{ DP } \\
\hline & & TI $\mathrm{PI}$ & NIS & PS TS & & \\
\hline $\begin{array}{l}\text { O nivel de segurança e estabilidade que the } \\
\text { oferece a empresa }\end{array}$ & & $3,1 \quad 1,5$ & 12,33 & $35,447,7$ & 4,23 & 0,95 \\
\hline $\begin{array}{l}\text { As relações que mantém com seus } \\
\text { companheiros de trabalho }\end{array}$ & V02 & $1,5 \quad 4,6$ & 4,65 & $53,835,4$ & 4,22 & 0,79 \\
\hline $\begin{array}{l}\text { As possibilidades de aprender e se desenvolver } \\
\text { profissionalmente }\end{array}$ & V03 & $1,5 \quad 4,6$ & 12,33 & $38,543,1$ & 4,20 & 0,89 \\
\hline A forma como lhe pagam & V04 & $4,6 \quad 6,2$ & 7,74 & $40,041,5$ & 4,08 & 1,09 \\
\hline $\begin{array}{l}\text { O respeito que lhe oferecem os companheiros } \\
\text { com os quais trabalha }\end{array}$ & V05 & $1,5 \quad 4,6$ & 12,34 & $49,232,3$ & 4,08 & 0,87 \\
\hline O respeito que lhe oferecem seus superiores & V06 & $3,1 \quad 9,2$ & 7,74 & $43,136,9$ & 4,05 & 1,03 \\
\hline e recebe por parte de seus superiores & V07 & $3,1 \quad 6,2$ & 10,84 & $46,233,8$ & 4,05 & 0,97 \\
\hline A segurança no emprego, frente ao futuro & V08 & $4,6 \quad 6,2$ & 15,43 & $30,843,1$ & 4,02 & 1,13 \\
\hline $\begin{array}{l}\text { ão de seu trabalho à sua formação e } \\
\text { profissionais }\end{array}$ & V09 & $3,1 \quad 4,6$ & 13,84 & $47,730,8$ & 4,02 & 0,93 \\
\hline A possibilidade de fazer amigos no trabalho & V10 & $4,6 \quad 4,6$ & 16,93 & $38,535,4$ & 3,98 & 1,05 \\
\hline $\begin{array}{l}\text { Os elogios que recebe quando obtém bons } \\
\text { resultados }\end{array}$ & V11 & $4,6 \quad 6,2$ & 21,53 & $30,836,9$ & 3,94 & 1,07 \\
\hline $\begin{array}{l}\text { O nível de conhecimentos e habilidades que } \\
\text { pode pôr em jogo no posto de trabalho }\end{array}$ & $\mathrm{V} 12$ & $0,010,8$ & 20,04 & $43,126,2$ & 3,90 & 0,93 \\
\hline $\begin{array}{l}\text { O reconhecimento que recebe por parte de seus } \\
\text { superiores quando merece }\end{array}$ & V13 & 7,710 & 10,84 & $44,626,2$ & 3,73 & 1,18 \\
\hline O salário que recebe & V14 & $7,7 \quad 15,4$ & 420,04 & $46,210,8$ & 3,39 & 1,11 \\
\hline O ambiente físico de seu posto de trabalho & $\mathrm{V} 15$ & $10,821,5$ & 523,12 & $29,215,4$ & 3,19 & 1,25 \\
\hline $\begin{array}{l}\text { As condições físicas no seu ambiente de } \\
\text { trabalho }\end{array}$ & V1 & $10, \varepsilon$ & 29 & $27,713,8$ & 3,17 & 1,20 \\
\hline $\begin{array}{l}\text { Os benefícios sociais que a empresa lhe oferece } \\
\text { (alimentação, plano de saúde...). }\end{array}$ & V & 2 & 18 & $27,7 \quad 10,8$ & 2,88 & 1,33 \\
\hline
\end{tabular}

As variáveis com pior avaliação (V14, V15, V16 e V17) se posicionaram no estrato intermediário, uma vez que todas tiveram médias próximas a 3,00. Assim, o ambiente (V15) e as condições físicas do local de trabalho (V16), com médias e desvios padrão de 3,19 $\pm 1,25$ e $3,17 \pm 1,20$, respectivamente, além do salário recebido $(\mathrm{V} 14,3,39 \pm 1,11)$, não podem ser considerados aspectos que contribuem para a satisfação com o 
trabalho, da mesma forma, não prejudiciais, assim foram avaliados como regulares pelos entrevistados. A pior avaliação da pesquisa coube aos benefícios sociais ( $\mathrm{V} 17,2,88 \pm 1,33)$, com média pouco abaixo de 3,00.

Os benefícios compreendem formas de salários indiretos, como vale alimentação, plano de saúde, auxílios em geral, entre outros. Esses aspectos foram considerados como parcial ou totalmente insatisfatórios por $43,1 \%$ (28) dos entrevistados.

Por outro lado, um amplo conjunto de aspectos foi favorável à satisfação com o trabalho e o que mais contribuiu para a satisfação foi a segurança e a estabilidade que o serviço público proporciona (V01). Essa assertiva obteve a maior média e o menor desvio padrão da pesquisa $(4,23 \pm 0,95)$, mostrando que a dispersão das respostas em torno da média foi muito baixa.

Com isso, a garantia de estabilidade foi a única assertiva que obteve avaliação satisfatória de 83,1\% (54) dos entrevistados, tendo sido um dos elementos mais valorizados pelos servidores entrevistados.

A estabilidade, em termos gerais, é uma questão controversa na administração pública. Adquirida após o representam

elementos cumprimento do período do estágio probatório de três anos (BRASIL, 1988, 1990), para Bresser-Pereira (1996), ela contribui para tornar o serviço público ineficiente e acomodado e acaba por desestimular os servidores que se empenham em seus cargos.

Por outro lado, diversos estudos (ALMEIDA; MEIRELES, 2015; PAULISTA, 2004; SALLES; NOGUEIRA, 2006; SILVA; BALACIANO, 2011) convergem ao considerar que a estabilidade é o principal atrativo para a carreira pública. A estabilidade, conforme Paula e Queiroga (2015), integra-se na satisfação contextual que, juntamente com outros aspectos do ambiente de trabalho, como a possibilidade de crescimento profissional, os relacionamentos com os supervisores, entre outros, são necessários para aumentar a motivação dos indivíduos na realização das tarefas.

Outras questões bem avaliadas pelos servidores foram os relacionamentos com os colegas no local de trabalho (V02, 4,22 $\pm 0,79$ ), o respeito dispensado por eles (V05, 4,08 $\pm 0,87$ ) inclusive pelas chefias (V06, 4,05 $\pm 1,03$ ), além das possibilidades de fazer amigos $(\mathrm{V} 10,3,98 \pm 1,05)$ e do apoio que 
recebem dos superiores imediatos (V07, 4,05 $\pm 0,97)$. Esses aspectos corroboram outros estudos realizados no serviço público (GOMES; QUELHAS, 2003; OLIVEIRA, 2009), que mostraram que as relações interpessoais são consideradas fortes influenciadoras na satisfação com o trabalho. Assim, ambientes caracterizados por relações interpessoais positivas normalmente apresentam maior cooperação e confiança. Nessa perspectiva, construir relações sociais implica promover benefícios sociais e emocionais que influenciarão positivamente tanto para a saúde, quanto para o bem-estar no local de trabalho. Um ambiente de trabalho no qual prevaleçam relações de amizade e companheirismo diminui o estresse e contribui para a produtividade no trabalho (MOHLER; BYRNE; CROPANZANO, 2004).

O aprendizado profissional (V03, 4,20 $\pm 0,89$ ), a adequação do trabalho aos interesses profissionais dos servidores (V09, 4,02 $\pm 0,93$ ) e o nível de conhecimentos e habilidades exigidos para a realização das tarefas $(\mathrm{V} 12, \quad 3,90 \pm 0,93)$ revelaram a satisfação com a realização das tarefas e quando combinadas com possibilidade de qualificação profissional revelaram aspectos satisfatórios do trabalho no local da pesquisa. Nesse sentido, a Nova Administração Pública trouxe para o serviço público maior esforço em qualificar os servidores (SILVA; MELLO, 2013). Assim, iniciativas como o Plano Nacional de Desenvolvimento Pessoal (PNDP), instituído pelo Decreto Lei no 5707, de 23 de fevereiro de 2006, colocaram em evidência a utilização de técnicas de gestão para fins de aperfeiçoamento de pessoal (BRASIL, 2006).

$O$ reconhecimento também foi valorizado como positivo no local da pesquisa, os elogios recebidos pelos bons resultados $(\mathrm{V} 11,3,94 \pm 1,07)$ e 0 reconhecimento por parte dos superiores hierárquicos (V13, $3,73 \pm 1,18$ ) foram percebidos como aspectos benéficos na instituição, sendo fatores motivacionais relevantes. Completaram os aspectos positivos a forma como o trabalho é pago (V04, 4,08 $\pm 1,13)$ e a segurança no emprego (V08, 4,02 $\pm 1,13$ ), as possíveis hipóteses para essa avaliação se prendem à regularidade no pagamento dos salários ao funcionalismo público federal e à perspectiva de futuro, sustentada na 
estabilidade e nas possibilidades de Observa-se, portanto, que os principais agentes promotores da satisfação no trabalho no local da pesquisa estão fundamentados em fatores extrínsecos como segurança e estabilidade, possibilidades de aprender e se desenvolver profissionalmente. Além da forma como os salários são pagos, há ainda

\subsection{ANÁLISE INFERENCIAL:}

A avaliação geral da satisfação (H1) foi feita com a utilização de estatística paramétrica pelo emprego do teste $\mathrm{T}$, aplicado tanto na forma para uma amostra, para testar se as respostas se posicionavam significativamente no estrato de satisfação $(>3,00)$, quanto na forma para a duas amostras independentes, para testar se as diferenças observadas entre os grupos de técnicos administrativos e professores eram significativas ou não.

$\mathrm{Na}$ avaliação geral, foi adotado o ponto intermediário da escala de Likert $(3,00)$ como referência para o teste, com isso, o principal mérito do teste $T$ foi possibilitar verificar se a população se comportava conforme qualificação

profissional.

a segurança frente ao futuro e o reconhecimento pelos bons resultados; em contraponto, constatou-se que as condições físicas do local de trabalho e os benefícios oferecidos poderiam ser melhorados e adequados às expectativas e necessidades dos servidores.

H1. Isso posto, o teste assumiu uma perspectiva unilateral à direita, e a regra de decisão para rejeitar $\mathrm{H} 1$ foi se $a^{\operatorname{sig} . \leq \frac{\alpha}{2}}$ e o sinal da estatística de teste fossem positivos $(\mathrm{t}>0)$. Considerando a escala utilizada na pesquisa, a hipótese $\left(\mathrm{HO}_{1}\right)$ assumiu a especificidade abaixo:

$H 1_{0}$ : $\mu \leq 3$, ou seja, não se pode afirmar que os entrevistados estejam satisfeitos com o trabalho no local da pesquisa.

$\mathrm{H} 11$ : $\mu>3$, ou seja, existem evidências estatísticas para afirmar que os entrevistados estão satisfeitos com o trabalho no local da pesquisa.

A condição de normalidade da distribuição amostral, exigência do 
teste $\mathrm{T}$, foi constatada pelo teste Destaca-se que esse teste foi feito mesmo considerando que o tamanho da amostra ( $\mathrm{n}=65>30)$ atendia ao Teorema do Limite Central (TLC) e a assimetria dos dados ser baixa $(0,297)$.

Verificados os pré-requisitos, a média geral das respostas foi de 3,80 , posicionando-se acima do valor de referência $(3,00)$, e o desvio padrão foi baixo $(0,62)$, indicando que a dispersão dos dados em torno da
Shapiro-Wilks

(p-valor $=0,138)$.

média era pequena. A Tabela 2 mostra que a média da satisfação dos servidores entrevistados

é significativamente superior a 3,00 (t $(65)$ $=10,355 ; p<0,01)$, sendo estimada com 95\% de confiança de se posicionar entre 3,65 e 3,96. Esse resultado possibilita rejeitar $\mathrm{H}_{1}$ e admitir que há evidências estatísticas para afirmar que os entrevistados estão satisfeitos no trabalho no local da pesquisa.

Tabela 2 - Teste T para avaliação da Satisfação com o Trabalho.

\begin{tabular}{lccccc}
\hline \multicolumn{1}{c}{ Distribuição $t$} & $\begin{array}{c}\text { Valor da } \\
\text { Significância }\end{array}$ & $\begin{array}{c}\text { Diferença } \\
\text { da média }\end{array}$ & \multicolumn{2}{c}{ Limites do intervalo de confiança } & \multirow{2}{*}{ H0 } \\
\hline Médias 10,355 & 0,000 & 0,854 & 0,650 & 0,960 & Rejeita \\
\hline 64 graus de liberdade & & Valor de referência $=3,00$ & Intervalo de confiança=95\% \\
\hline Fonte: Pesquisa de campo.
\end{tabular}

Estabelecida a condição geral de satisfação com 0 trabalho, a investigação se concentrou em identificar se havia diferença significativa na satisfação entre os dois grupos de trabalhadores professores ( $\mathrm{n} 1=34)$ e técnicos administrativos (n2=31). Essa investigação foi feita considerando a média amostral de cada grupo, novamente com a utilização do teste $T$, porém na forma para duas amostras independentes. $\mathrm{O}$ valor definido para determinar a região crítica de aceitação e rejeição de $\mathrm{H} 2$ foi de $\alpha=0,05$ (bilateral).
Os pré-requisitos do teste foram verificados e atendidos, uma vez que: (1) as variáveis estavam em escala intervalar; (2) havia normalidade nas distribuições amostrais constatadas pelo teste Shapiro Wilks (SW), tanto para professores ( $p$-valor $=0,561)$, quanto para os técnicos administrativos ( $p$-valor $=0,392$ ); e (3) a homogeneidade das variâncias populacionais foi verificada pelo teste de Levene ( $F=1,090 ; p$-valor $=0,300)$, evidenciando que as amostras foram extraídas de populações com 
variâncias iguais, assim utilizou-se o hipótese (H2). Considerando as diferenças entre os grupos, a hipótese assumiu as seguintes especificidades: H20: $\mu_{1}=\mu_{2}$, ou seja, não se pode afirmar que existem diferenças nas médias das respostas de professores e técnicos administrativos quanto à satisfação com o trabalho.

$H 2_{1}: \quad \mu_{1} \neq \mu_{2}$, ou seja, existem evidências para afirmar que as médias das respostas de professores e teste $\mathrm{T}$ homocedástico para testar a técnicos administrativos são diferentes quanto à satisfação com o trabalho.

Apesar de as médias das respostas dos professores $(3,67 \pm 0,67)$ e técnicos administrativos $(3,95 \pm 0,54)$ serem diferentes, observa-se na Tabela 3, que ambos os grupos manifestaram satisfação com 0 trabalho, uma vez que as médias das respostas foram superiores ao ponto central da escala $(3,00)$.

Tabela 3 - Teste T para avaliação da satisfação com o trabalho de professore $(n 1=34)$ e técnicos administrativos ( $\mathrm{n} 2=31)$

\begin{tabular}{|c|c|c|c|c|c|c|c|}
\hline \multirow{2}{*}{ Variável } & \multicolumn{2}{|c|}{ Professores (n1) } & \multicolumn{2}{|c|}{ Técnicos (n2) } & \multirow{2}{*}{ Teste T } & \multirow{2}{*}{$\begin{array}{l}\text { Valor da } \\
\text { Significância }\end{array}$} & \multirow{2}{*}{$\mathrm{H} 2 \mathrm{O}$} \\
\hline & Média & $\mathrm{DP}$ & Média & DP & & & \\
\hline $\begin{array}{c}\text { Média } \\
\text { amostral }\end{array}$ & 3,67 & 0,67 & 3,95 & 0,54 & $-1,815$ & 0,074 & Aceita \\
\hline
\end{tabular}

Portanto, os resultados do teste $\mathrm{T}$

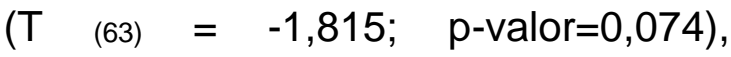
superior a $\alpha=0,05$ (bilateral), não permitem rejeitar $\mathrm{H} 20$, admitindo que não há diferença significativa na satisfação com o trabalho entre os

\section{CONCLUSÕES}

O estudo buscou identificar os elementos no ambiente de trabalho que contribuem para a satisfação com - trabalho de servidores públicos lotados em uma Instituição Federal de Ensino Superior (IFES). Os resultados grupos identificados. Desta forma, considera-se que as diferenças encontradas ocorreram ao acaso e se constata que a atividade não influencia na satisfação com o trabalho no local da pesquisa. 
que proporcionam maior satisfação são as relacionadas com a estabilidade, segurança e relacionamento com os colegas. Intermediariamente, posicionaram-se os aspectos físicos, as instalações e a estrutura do ambiente de trabalho e, com pior avaliação, os benefícios sociais recebidos, porém esse aspecto, apesar de ser 0 de pior avaliação, posicionou-se muito próximo aos demais, posicionando-se no centro da escala.

No que tange às hipóteses, foi rejeitada a hipótese de que não se pode afirmar que existe satisfação entre os trabalhadores e aceita a que afirma inexistirem diferenças na satisfação entre as categorias de docentes e técnicos administrativos,

\section{REFERÊNCIAS}

ALMEIDA, D. R.; MEIRELES, A. S. Satisfação e Trabalho: uma análise do grau de satisfação dos servidores em estágio probatório da UFBA. In: Colóquio Internacional de Gestão Universitária, 2015, Mar Del Plata. Anais...Argentina, 2015. Disponível em: <https://repositorio.ufsc.br/handle/123456789/136146>. Acesso em 01 set. 2016.

BARBETTA, P. A. Estatística aplicada a ciências sociais. Florianópolis: Editora da UFSA, 2014.

BARBOZA, J. R. A satisfação profissional e a cultura organizacional: uma análise a partir do modelo ASH no Centro Federal de Educação Tecnológica do Rio Grande do Norte. Natal, 2008. Dissertação (Mestrado em Administração) - Universidade Federal do Rio Grande do Norte, Programa de Pós-Graduação em Administração, Natal, 2008.

BERTOLDO, M. R. A satisfação dos clientes internos e o comprometimento organizacional: um estudo à luz do modelo ASH nas indústrias alimentícias do Rio Grande do Norte. Natal, 2006. Dissertação (Mestrado em Administração) - 
Universidade Federal do Rio Grande do Norte, Programa de Pós-Graduação em Administração, Natal, 2006.

BRASIL. Constituição da República Federativa do Brasil, 05 de outubro de 1988. Disponível

em: $<$ http://www.planalto.gov.br/ccivil 03/constituicao/constitui\%C3\%A7ao.htm>. Acesso em 10 out. 2016.

BRASIL. Decreto Federal $n^{\circ}$ 5707, de 23 de fevereiro de 2006. Institui a Política e as Diretrizes para o Desenvolvimento de Pessoal da administração pública federal direta, autárquica e fundacional, e regulamenta dispositivos da Lei n 8.112, de 11 de dezembro de 1990.

BRASIL. Lei n. 8.112, de 11 de dezembro de 1990. Dispõe sobre o regime jurídico dos servidores públicos civis da União, das autarquias e das fundações públicas federais. Disponível em: <http://www.planalto.gov.br/ccivil_03/leis/L8112cons.htm>. Acesso em 10 out. 2016.

BRESSER-PEREIRA, L. C. Da administração pública burocrática à gerencial. Revista do Serviço Público, Brasília, v. 47, n. 1, jan. /abr. 1996. Disponível em: $<$ http://www.bresserpereira.org.br/papers/1996/95.AdmPublicaBurocraticaAGerencial .pdf >. Acesso em: 10 set. 2016.

BRUM, T. M. M.; MARTINELLI, S. G.; WAECHTER, L.; MÜLLER, L.; JÑANA, G. M. A influência da socialização e integração na satisfação dos servidores públicos: um estudo de caso em uma organização pública. Desafio On-line, v.2, n. 1, p. 465-486. jan. I abr. 2014. Disponível em: <http://seer.ufms.br/index.php/deson/article/view/1132>. Acesso em: 04 mar. 2017.

BRUNI, A. L. SPSS aplicado a pesquisas acadêmicas. São Paulo: Atlas, 2013.

CAMPBEL, A; CONVERSE, P.E.; RODGERS, W. L. The quality of American Life: perceptions, evaluations, and satisfaction. New York: Sage, 1976.

DAVIS, R. Unionization and work attitudes: how union commitment influences public sector job satisfaction. Public Administration Review, v. 73, n. 1, p. 74-84, jan. / fev. 2013. Disponível em: <http://onlinelibrary.wiley.com/doi/10.1111/j.15406210.2012.02609.x/abstract>. Acesso em: 03 mar. 2017.

DUTRA, J. S. Gestão de pessoas articulada por meio de competências. In: TEIXEIRA, H. J.; BASSOTTI, I. M.; SANTOS, T. S. (Org.). Contribuições para a gestão de pessoas na administração pública. São Paulo: FIA/USP, 2013. Disponível em: $\quad<$ http://perfilmotivacional.com.br/estilos-comportamentais-dos-profissionais-daarea-de-gestao-de-pessoas-um-estudo-exploratorio/>. Acesso em 05 out. 2016.

FRASER, T. M. Work, fatigue, and ergonomics. In: Introduction to industrial ergonomics: a textbook for students and managers (online). Toronto: Wall and Emerson, 1996. Disponível em <http://www.wallbooks.com/source/fraser.htm.> Acesso em 25 set. 2016. 
GIL, A.C. Métodos e técnicas de pesquisa social. São Paulo: Atlas, 2014.

GOMES, A. A. E.; QUELHAS, O. L. G. Motivação dos recursos humanos no serviço público: um estudo de caso sob dois ângulos teóricos. Revista Eletrônica de Administração, v. 9, n. 5, set. / out. 2003. Disponível em: <http://www.read.ea.ufrgs.br/edicoes/pdf/artigo 30.pdf>. Acesso em 14 set. 2016.

HAIR Junior, J. F. et al. Análise multivariada de dados. Porto Alegre: Bookman, 2009.

HAMPTON, D. R. Administração contemporânea. São Paulo: Makron Books, 1992.

KJELDSEN, A.M.; HANSEN, J.R. Sector differences in the public service motivationjob satisfaction relationship: exploring the role organizational characteristics. Review of Public Personnel Administration, Online First, p. 1-25, fev. 2016. Disponível em: $<$ http://journals.sagepub.com/doi/abs/10.1177/0734371X16631605?journalCode=rop a $>$. Acesso em: 03 mar. 2017.

LOCKE, E. A. What is job satisfaction? Organizational Behaviour Human Performance. v. 4, n.4, p. 309-336, 1969. Disponível em: $<$ http://www.sciencedirect.com/science/article/pii/0030507369900130>. Acesso em 25 ago. 2016.

MALHOTRA, N. K. Pesquisa de marketing: uma orientação aplicada. São Paulo: Bookman, 2011.

MARQUES, A. L.; BORGES, R.; REIS, I.C. Mudança organizacional e satisfação no trabalho: um estudo com servidores públicos do estado de Minas Gerais. Revista de Administração Pública, v. 50, n. 1, p. 41-58, jan. / fev. 2016. Disponível em: $<$ http://www.scielo.br/pdf/rap/v50n1/0034-7612-rap-50-01-00041.pdf >. Acesso em: 03 mar. 2017.

MARQUEZE, E. C.; MORENO, C. R. C.. Satisfação no trabalho: uma breve revisão. Revista Brasileira de Saúde Ocupacional, v. 30. n. 112, p. 69-79, 2005. Disponível em:<http://www.scielo.br/pdf/rbso/v30n112/07.pdf>. Acesso em: 10 out. 2016.

MELEIRO, A. R.; SIQUEIRA, M. M. M. Os impactos do suporte do supervisor e de estilos de liderança sobre o bem-estar no trabalho. In: Encontro da Associação Nacional de Pós-Graduação em Administração, 2005. Brasília. Anais... Brasília: ANPAD, 2005.2 Disponível em: $<$ http://www.anpad.org.br/diversos/trabalhos/EnANPAD/enanpad 2005/EOR/2005 E ORB992.pdf>. Acesso em 18 set. 2016.

MELLO, M. L. B. C.; AMÂNCIO FILHO, A. A. A gestão de recursos humanos em uma instituição pública brasileira de ciência e tecnologia em saúde: o caso Fiocruz. Revista de Administração Pública, v. 44, n. 3. p. 613-636, 2010. Disponível em: <http://www.scielo.br/pdf/rap/v44n3/04.pdf>. Acesso em: 15 set. 2016.

MOHLER, C. J..; BYRNE, Z. S.; CROPANZANO, R. Emotional exhaustion, work relationships and health effects on organizational outcomes. In: The 19th Annual Rev. Cereus, v. 9, n. 1, p.57-75, jan-abr./2017, UnirG, Gurupi, TO, Brasil. 
Conference of the Society for Industrial and Organizational Psychology, Chicago, 2004.

Disponível em: $<$ https://www.researchgate.net/publication/228510952 Emotional Exhaustion Work Relationships and Health Effects on Organizational Outcomes>. Acesso em 15 out. 2016.

PAULA, A. P. V.; QUEIROGA, F. Satisfação no trabalho e clima organizacional: a relação com autoavaliações de desempenho. Revista Psicologia: Organizações e Trabalho, n. 15, v. 4, p. 363 - 373, out. / dez. 2015. Disponível em: $<$ http://pepsic.bvsalud.pdf/rpot/v15n4/v15n4a04.pdf>. Acesso em 14 out. 2016.

PAULISTA, J. E. S. A Motivação dos servidores públicos federais, no âmbito dos Ministérios, frente às premissas de valorização firmadas no Plano Diretor da Reforma do Aparelho do Estado, de 1995, e às consequentes ações de gestão de recursos humanos. 2004. 155 f. Dissertação (Mestrado em Administração Pública) Fundação Getúlio Vargas, Rio de Janeiro, 2004.

PINTO, C. F. P; SILVA, F. M. Gestão de pessoas na administração pública brasileira: uma análise sobre sua evolução histórica e 0 atual contexto em transformação. Revista Eletrônica do Tribunal de Contas do Estado - RS, v. 1, n. 2, p. 1-16, jul. /dez, $2015 . \quad$ Disponível em: $<$ http://revista.tce.rs.gov.br/index.php/revtce/article/view/14>. Acesso em 17 out. 2016.

QUIJANO, S. D.; NAVARRO, J. ASH (Auditoria del Sistema Humano), los modelos de calidad y la evaluación organizativa. Revista de Psicología General y Aplicada, v. 52, $\quad$ p. $301 \quad$ - $\quad 328 . \quad 1999 . \quad$ Disponível em: $<$ https://dialnet.unirioja.es/descarga/articulo/2498353.pdf>. Acesso em: 10 jun. 2016.

ROBBINS, S.P. Comportamento organizacional. São Paulo: Prentice Hall, 2014.

SALLES, D. M. R.; NOGUEIRA, M. G. Carreiras no serviço público federal: antigos dogmas, novas perspectivas. In: BALASSIANO, M.; COSTA, I. S. A da (Orgs.). Gestão de Carreiras: dilemas e perspectivas. Rio de Janeiro: Atlas, 2006.

SANT'ANNA, A. S.; MORAES, L. F.; KILIMNIK, Z. M. Competências Individuais requeridas, modernidade organizacional e satisfação no trabalho: uma análise de organizações mineiras sob a ótica de profissionais da área de administração. In: Encontro da Associação Nacional de Pós-Graduação em Administração, 2002, Atibaia. Anais... Brasília: ANPAD, 2002.

SILVA, F. M.; MELLO, S. P. T. A implantação da gestão por competências: práticas e resistências no setor público. Revista Eletrônica de Administração e Turismo, n. 1, v. 2, p. $110-127$, jan. / jun. 2013. Disponível em: $<$ https://periodicos.ufpel.edu.br/ojs2/index.php/AT/article/view/1959/2339>. Acesso em 16 out. 2016.

SILVA, J. R.; BALACIANO, M. Construção dos Processos Pessoais de Carreiras no Setor Público no Contexto Social Contemporâneo. In: Encontro da Associação Nacional de Pós-Graduação em Administração, 2011. Brasília. Anais... Brasília: 
ANPAD,

2011.

Disponível

em:

$<$ http://www.fucape.br/ public/producao cientifica/2/moises.pdf > Acesso em 10 set. 2016.

TEIXEIRA, H. J.; SALOMÃO, S.M. Visão sistêmica e gestão de pessoas. In: TEIXEIRA, H; J.; BASSOTTI, M.; SANTOS, T.S. Contribuições para a gestão de pessoas na administração pública. São Paulo: FIA/USP, 2013.

Recebido em: 10/12/2016.

Aprovado em:14/03/2017. 